\title{
Analysis Effectiveness and Stability of Corporate Marketing Network
}

\author{
Qin xiaohong* \\ School of Economics and Business \\ xijing University \\ Xi'an,China \\ Qinxiaohong12@sohu.com
}

\author{
Ma jing \\ School of Economics and Business \\ xijing University \\ Xi'an,China \\ Qinxiaohong12@sohu.com
}

\begin{abstract}
Actual corporate marketing network tend to have a large and complex network structure, which affect the operation of enterprises and even dominate marketing network. Based on complex network theory, stability and effectiveness of the definition of corporate marketing network, the establishment of the enterprise network marketing raw form model to analyze effective and stable corporate marketing network features.
\end{abstract}

Keywords-Corporate marketing network; endogenous model; complex network ;effectiveness; stability

\section{INTRODUCTION}

Corporate marketing network is the between members interrelated and interact to formed the network structure, this structure dominates the operation of enterprises and even affect the marketing network operating[1]. Actual corporate marketing network tend to have a large and complex network structure, however, it is assumed that the classical economic theory of corporate marketing network has a simple network structure completely or star-connected, so in previous studies in economics, corporate marketing network structure neglected, perhaps one of the reasons the classical economic theory of real enterprise networks often lack the ability to explain the marketing [2].

1999 BA made a complex network of landmark studies [3], they reveal a lot of practical network connection has a scale-free this striking results. Excited this research, people began to pay attention network structure in the economic field, to carry out a number of empirical studies on the economic structure of the network. Results of the study show: many aspects of people in the economic field was also observed network scale-free BA described, while the nature of these economic networks also have a small world and so on. 1996 Jackson and Wolinski first used game theory to study the formation of the way economic networks, trying to come from microeconomic principles to explain why economic network formation, while introducing economic concepts of network stability and effectiveness [4]. It is natural to ask the question of how corporate marketing network is formed.

This paper proposes the definition of stability and effectiveness of corporate marketing network, the establishment of conditions for the formation of student enterprise marketing network model to analyze the effective marketing network and a stable marketing network to meet. And numerical column will be described.

\section{CORPORATE MARKETING NETWORK AVAILABILITY AND STABILITY DEFINITION}

\section{A. Corporate marketing network representation mark}

Consider a finite individuals (to participate in corporate marketing activities of organizations or individuals) consisting of marketing network, with nodes represent participation in corporate marketing activities of organizations or individuals (marketing network member). If the two nodes $i$ and $j$ between product supply and demand if there is relationship or transaction information exchange relations, the establishment of an even edge which denoted $e_{i j}$, while the right is used $w_{i j}$ to indicate, with a trade volume of the weight or information flow between the nodes $i$ and $j$ to represent, so corporate marketing network to abstract complex network .Use $V=\{1,2, \quad, n\}$ to representation all collection nodes of corporate marketing network, all nodes number denoted $N$; set $G=\left\{e_{i j} \mid i, j \quad G\right\}$ record network all side remember that all side remember is $e_{i j} \quad G$. all side remember denoted $M$.

Consider a subset $S \quad V$ of the set of nodes, we can define the network $\left.G\right|_{S}$, it satisfies the conditions $\left.\forall e_{i j} \quad G\right|_{S} \quad i, j \quad S$ and $\left.G\right|_{S} \quad G .\left.G\right|_{S}$ is limited to nodes $S$ the subset of network. Then define a fully connected network $G^{V}=\left\{e_{i j} \mid i, j \quad V\right\}$, all the nodes of the network that is connected to each other, fully connected network $G^{V}$ representation exists product supply and demand transaction or information exchange between corporate sales and marketing network in any organization or individual, then there must be $G G^{V}$. Since we note $G$ in the collection of $G^{V}$ all subsets, that this is all $G \quad G^{V}$ for all possible network composed by 
a set of nodes $V$. In addition, we use $G^{\phi}$ to indicate an empty network connection that all its nodes are isolated, that is, there is no corporate marketing network.

\section{B. Corporate marketing network availability and stability}

The formation of enterprise marketing network is the result of market economy organization or individual (economic actors) rational decision-making behavior, which can be considered to form a network of corporate marketing organization or endogenous process of individual economic activity, can be used similar Nash Equilibrium as a starting point for analysis of corporate marketing network formation process modeling [5]. By giving each enterprise marketing network nodes $i$ (node represents corporate marketing organization or a network) function $u_{i}: C \quad R$ to make some kind of reward for each organization or individual can make a rational policy choice, and thereby induce some across the entire network species total utility function. If the marketing network in the presence of a pair of late with two nodes connected by an edge, wherein at least one node is considered to delete this side will be better for him; or if there is a pair of two individuals not connected by an edge, they are consider adding an edge will both be better for both of them; then the business marketing network thus formed is unstable otherwise it is stable pairs. This shows that, during the formation of corporate marketing network nodes in why some transactions or the establishment of product supply and demand relationship and exchange of information to other nodes and maintain this relationship, and some have established product supply and demand information exchange transaction or node can not sustain this relationship species relationship. Effective business marketing network referred to when business marketing network to achieve optimal total utility function, and effectiveness is used to measure of the formation of whether an indicator of optimality. Without loss of generality, so that the total effect of corporate marketing network to meet the normalization conditions: $u_{i}\left(G^{\phi}\right)=0$, So the total utility function of corporate marketing network $G$ is all nodes utility total, namely:

$$
U(G)=u_{i V} u_{i}(G)
$$

Definition 1: In corporate marketing network $G G^{V}$, if there is no one pair of network members want to build product supply and demand transaction or information exchange relations, but also did not want to disconnect any network members and other network members established product supply and demand transaction or information exchange relations, then the marketing network $G$ called stable, stable marketing network to meet the following two conditions:

$$
\begin{aligned}
& \text { (1) } \forall e_{i j} G \text {, Then } u_{i}\left(G-e_{i j}\right) \quad u_{i}(G) \text { and } \\
& u_{j}\left(G-e_{i j}\right) \quad u_{j}(G)
\end{aligned}
$$

$$
\text { ( } 2 \text { ) } \forall e_{i j} G \text {, in case } u_{i}\left(G+e_{i j}\right)>u_{i}(G) \text {, }
$$

certainly has $u_{j}\left(G+e_{i j}\right)<u_{j}(G)$

Definition 1 Description: In a stable business marketing network in neither one willing to Wang Yuan disconnect between their existing trading relationship between supply and demand of products or information exchange relations, nor a pair of network members are willing to build products between them supply and demand relationship or transaction information exchange relations.

Decree:

$$
u_{e_{i j}}=\begin{aligned}
& u_{i}(G)-u_{i}\left(G-e_{i j}\right), e_{i j} \quad G \\
& u_{i}\left(G+e_{i j}\right)-u_{i}(G), e_{i j} \quad G
\end{aligned}
$$

The formula gives the utility value between the node $i$ and the node $j$ product supply and demand information exchange trading relationship or the relationship obtained. So for is a stable marketing network situation, there were $e_{i j} G$, and there was $u_{e_{i j}} \quad 0$, then $u_{e_{i j}} G, u_{e_{i j}}$ and $u_{e_{j i}}$ both have at least one less than zero. Wherein the utility between $u_{e_{i j}}$ and $u_{e_{i}}$ represent the node $i$ and node $j$ product supply and demand relationship or transaction information exchange relations were obtained respectively.

Definition 2: If the utility value of corporate marketing network $G \quad G^{V}$ network members consisting of the same marketing network is the largest, then $G$ is called the effective marketing network marketing network. Effective marketing network to meet the following conditions:

$$
U\left(G^{\prime}\right) \quad U(G), \forall G^{\prime} \quad G
$$

Effective corporate marketing network is not unique. For example the set of enterprise marketing network $G$ utility function for each network members depends only on contact with the other circumstances of the members but nothing to do with the specific network members, all with the topology of the same marketing network marketing network $G$ and $G^{\prime}$ has the same total utility.

\section{ENDOGENOUS MODEL OF CORPORATE MARKETING NETWORK}

\section{A. Endogenous model}

The members of enterprise Marketing network with other network members around it due to supply and demand transaction or information exchange product and have contact, and derive some utility as income [6]. It is assumed that, if the utility is an indirect product of supply and demand between the two members transaction or information exchange and contact, then it will get connected with the distance between them diminishes. At the same time as each other members has to maintain relationships with its direct product supply and demand transaction or information exchange and contact between, which will also take it to maintain the normal operation of 
this relationship and to pay certain costs. And each of the network members need to maintain relationships with other network members and it happens direct product supply and demand transaction or information exchange and contact between the cost paid, without the need to maintain its direct marketing network does not occur in product supply and demand transaction or relations of exchange of information and other network members to pay any fees. Members if both product supply and demand information exchange transaction does not occur directly or indirectly linked to the direct product of supply and demand can never happen transactions or changes to supply and demand information exchange transactions or exchange of information and to obtain a direct product of a positive utility value, they will product supply and demand will increase in direct transaction or information exchange occurs;And if a particular utility to maintain the members found it a direct product of supply and demand transaction or information exchange relationships with other network members are derived from them is negative, he will break the direct communication with the network members supply and demand transaction or product information. Each network members through this growing disconnect evolution or linked, if this will tend to reach some kind of stable marketing network, the network is thus formed is regarded as the endogenous formation of corporate marketing network [7].Specifically, the corporate marketing network $G$ at the moment $t$, we assume that the network paid member $i$ has the following functions:

$$
u_{i}=\underset{j N\left(G_{t}\right)\{\{i\}}{w_{i j}} \lambda_{i j}^{d(i, j)}-{ }_{j N_{i}\left(G_{t}\right)} c_{i j}
$$

Where $w_{i j}$ reflects the degree of interaction between the network members $i$ and $j$, in which equal weights of edges between network members $i$ and $j$. The parameters $\lambda_{i j}(0,1)$ is attenuation coefficient with $i$ and $j$ between distance. Here, if the network member $i$ and $j$ do not occur direct and indirect product supply and demand transaction or information exchange, the $d(i, j)=$, so the network will not have any impact on member $i$ (utility zero) for network members $j$. The parameter $c_{i j}>0$ is the cost of network member $i$ and network members $j$ in order to maintain product supply and demand transaction or information exchange relationship between them to be paid, and $c_{i j}=c d(i, j)$, where $d(i, j)$ is the spatial distance between the network members $i$ and $j$, here, $d(i, j)$ is defined shortest marketing channels of network member $i$ and network members $j$. It can be seen: the definition of parameters are linked to two network members symmetrical about that, $w_{i j}=w_{j i}, \lambda_{i j}=\lambda_{j i}$ and $c_{i j}=c_{j i}, \forall i j \quad G_{t}$, this link between the two companies marketing network members are mutually related.

According to the above definition, the enterprise marketing network model is formed by students of the emerging network architecture evolution will depend on the utility function (3) formula given, of course, we can also promote the above utility function to a more general case, in order to generate the desired more different topologies marketing network.

The paper will not be concerned about the formation of a stable network of corporate marketing dynamic process, the first to examine the definition of one of the companies marketing network. Here we will study: meet the definition of stability according to corporate marketing network exist? If present, they will be what structure? Its effectiveness and how? The following will examine these issues.

\section{B. Effective and stable corporate marketing network features}

Theorem 1: $\forall e_{i j} \quad G$, effective corporate marketing network marketing network formed by the endogenous model, there are:

When $c_{i j}>\lambda_{i j}+\frac{n-1}{2} \lambda_{i j}^{2}$, the empty network is the only effective corporate marketing network. Indicates the absence of the corporate network, the company's products can not be sold on the market;

When $\lambda_{i j}-\lambda_{i j}^{2}<c_{i j}<\frac{n-1}{2} \lambda_{i j}^{2}$, the star structure marketing network is the only effective marketing network. Indicate the presence of a central network marketing network member, the remaining networks are occurring product supply and demand transaction or information exchange through the network;

When $c_{i j}<\lambda_{i j}-\lambda_{i j}^{2}$, the fully connected marketing network is the only effective network. Product supply and demand shows that there are transactions or any exchange of information between network members.

Certificate: (1) Examine any marketing network having a communication $m \quad a \quad 1$ edges, ) 。(Which $a-1$ is composed of corporate marketing network connectivity required minimum transaction or product supply and demand have a direct exchange of information on the number of network members). Network members $i$ and $j$ utility gained from the transaction or product supply and demand relationship in the exchange of information will come from two parts: The utility is part of the exchange of information provided by the direct relationship between supply and demand of products or transactions, a value of $2 m \lambda_{i j}, \forall i, j$, the multiplier factor 2 is due to supply and demand for each product transaction or information exchange relations with two network members mutual benefits; Another part of the utility is indirectly $[a(a-1) / 2]-m$ pair of network 
members and other product supply and demand transaction or information exchange relations provided, the length of these marketing channels is at least 2 , so its value is not greater than $2\{[a(a-1) / 2]-k\} \lambda^{2}$. For the cost, because the transaction or product supply and demand information exchange network of member $i$ and $j$ together are maintained by them, the cost of at least $2 c_{i j}, m$ edges of all the costs of the $2 m c_{i j}, \forall i, j$. So the upper bound of the total utility companies marketing network has the following estimate:

$B_{m}=2 m \lambda_{i j}+2 \frac{a(a-1)}{2}-m \quad \lambda_{i j}^{2}-2 m c_{i j}, \forall i, j$

$$
\text { which is: } U(G) \quad B_{m}, \forall G \quad\{G \mid G \quad m\}
$$

When take $k=a-1$ there is:

$$
B_{a-1}=2(a-1) \quad \lambda_{i j}+\frac{a-2}{2} \lambda_{i j}^{2}-c_{i j}, \forall i, j
$$

Corporate marketing network star topology just reached this upper bound, the upper bound on the formula for the number of edges of corporate marketing communication network $m=a-1$ can be achieved supremum.

Thus, we found that for corporate marketing communication network are:

$$
B_{m+1}-B_{m}=2 \lambda_{i j}-2 \lambda_{i j}^{2}-2 c_{i j}, \forall i, j, \forall m \quad a \quad 1
$$

(6)

The formula does not depend on $m$, which indicates that: when $c_{i j}>\lambda_{i j}-\lambda_{i j}^{2}, \forall i, j$, rational network members have no interest to maintain product supply and demand relationships or transactions with the exchange of information between networks $a-1$ others, can be seen from (5), when $c_{i j}>\lambda_{i j}+(a-2 / 2) \lambda_{i j}^{2}$, there is $B_{a-1}<0$. When the number of sides $m a 1$, apparently unconnected total utility marketing network does not exceed the total utility the same side of a connected network number (because the same connection cost the latter to the former, but the latter has a more direct connection side). When the number of sides $m \quad a 1$, the marketing network node must exist in isolation, so the condition $c_{i j}>\lambda_{i j}, \forall i, j$, the inevitable push marketing network total utility is negative. So, then by $U\left(G^{\phi}\right)=0$ certified that conclusion.

( 2 ) Consider a star-marketing network $G^{*}$, the central node is $i$. Since $c_{i j}<\lambda_{i j}+\frac{a-2}{2} \lambda_{i j}^{2}, \forall i, j$, to remove any one side $e_{i j}$ from $G^{*}$ will make network total utility change $2 c_{i j}-\lambda_{i j}-\frac{a-2}{2} \lambda_{i j}^{2}<0$, so $G^{*}$ is more effective than $\left(G^{*}\right)^{-}$. And because $c_{i j}>\lambda_{i j}-\lambda_{i j}^{2}$, from $G^{*}$ to add any edge $e_{j h}$ will make the network forms a triangle $\left(G^{*}\right)^{+}$ring, so the total utility change $2\left(\lambda_{i j}-\lambda_{i j}^{2}-c_{i j}\right)<0$, so $G^{*}$ still more effective than $\left(G^{*}\right)^{+}$.According to (1) demonstrate the results for formula (5) we can see, when $\lambda_{i j}-\lambda_{i j}^{2}<c_{i j}<\lambda_{i j}+\frac{a-2}{2} \lambda_{i j}^{2}, U\left(G^{*}\right)=B_{a-1}>0$.

And when the sides number $m<a-1$, there $G$ must be several mutually communicating sub-marketing network connection (including some isolated nodes). So we can see from the foregoing discussion, each connected sub network same total utility of these nodes consisting of a star child marketing network is the largest. There are two non-contiguous nodes $n_{1}$ and $n_{2}$ are a star child marketing network $G^{\prime}$ and $G^{\prime}$, the corresponding total utility values is:

$U^{\prime}=U^{\prime}\left(G^{\prime}\right)=$

$$
\begin{aligned}
& 2\left(n_{1}-1\right) \lambda_{i j}+\frac{n_{1}-2}{2} \lambda_{i j}^{2}-c_{i j}, \forall i, j \\
& U^{\prime \prime}=U^{\prime \prime}\left(G^{\prime \prime}\right)= \\
& 2\left(n_{2}-1\right) \lambda_{i j}+\frac{n_{2}-2}{2} \lambda_{i j}^{2}-c_{i j}, \forall i, j
\end{aligned}
$$

For the validity of the marketing network, we only think of their total utility are non-negative cases, as a connecting link of the two sub-central node marketing network, the two sub-marketing network connectivity obtained $\bar{G}$, it's total utility as follows:

$$
\begin{aligned}
U^{\prime}+U^{\prime \prime} & U(\bar{G}) \quad z\left(n_{1} 1\right)+\lambda_{i j} \frac{n_{1}-2}{2} \lambda_{i j}^{2} c_{i j} \\
+ & 2\left(n_{2}-1\right) \lambda_{i j}+\frac{n_{2}-2}{2} \lambda_{i j}^{2}-c_{i j} \\
+ & 2 \lambda_{i j}+2\left(n_{1}+n_{2}-2\right) \lambda_{i j}^{2} \\
+ & 2\left(n_{1}-1\right)\left(n_{2}-1\right) \lambda_{i j}^{3}-2 c_{i j}
\end{aligned}
$$

Formula (9) $\lambda_{i j}^{3}$ replace with $\lambda_{i j}^{2}$,we can obtained Formula (10):

Wherein $\bar{n}=n_{1}+n_{2}$, that is the star marketing network non-negative utility value by the above method to connect more effectively. From above, we can see that the star structure $G^{*}$ in all the structures is have largest and positive total utility, therefore, $G^{*}$ is the only effective marketing network structure.

(3) For not fully connected marketing network $G$, then there can be added to the side so that the network expansion is $G^{+}$. In this case, the total utility value will 
increase at least $2\left(\lambda_{i j}-\lambda_{i j}^{2}-c_{i j}\right)$, as $c_{i j}<\lambda_{i j}-\lambda_{i j}^{2}$, the network is always desirable to increase the edge until the network becomes fully connected network so far.

$$
\begin{aligned}
U^{\prime}+U^{\prime \prime} & <2\left(n_{1}-1\right) \lambda_{i j}+\frac{n_{1}-2}{2} \lambda_{i j}^{2}-c_{i j} \\
& +2\left(n_{2}-1\right) \lambda_{i j}+\frac{n_{2}-2}{2} \lambda_{i j}^{2}-c_{i j} \\
& +2 \lambda_{i j}+2\left(n_{1}+n_{2}-2\right) \lambda_{i j}^{2} \\
& +2\left(n_{1}-1\right)\left(n_{2}-1\right) \lambda_{i j}^{2}-2 c_{i j} \\
& =2(\bar{n}-1) \lambda_{i j}+\frac{-2}{2} \lambda_{i j}^{2}-c_{i j} \\
& =B_{\bar{n}-1}
\end{aligned}
$$

Theorem 2: : (Stability) $\forall e_{i j} G$, stable marketing network formed by the endogenous model, there are:

Stable marketing network at most only one non-empty sub-network;

When $c_{i j}<\lambda_{i j}-\lambda_{i j}^{2}$, then fully connected network $G^{v}$ is only stable network;

When $\lambda_{i j}-\lambda_{i j}^{2}<c_{i j}<\lambda_{i j}$, then star network $G^{*}$ is a pair of stable network, but it is not necessarily the only stable network;

When $c_{i j}>\lambda_{i j}$, then any non-empty and stable marketing network each node it's degree at least 2 , and this marketing network is invalid.

By contradiction to proof (1). Hypothesis a stable marketing network has two or more sub-network connectivity. Let $u_{i j}$ representation utility from the side $e_{i j} \quad$ of node $i$ : If $i j G$, then $u_{i j}=u_{i}\left(G+e_{i j}\right)-u_{i}(G)$; if $e_{i j} G$, then $u_{i j}=u_{i}(G)-u_{i}\left(G-e_{i j}\right)$. Consider the case $e_{i j} G$, then $u_{i j}>0$. Let side $f l$ and side $i j$ is not connected to another division within the communication, so $u_{l j}>u_{i j} \quad 0$. This is because if you add a side $l j$, Node $l$ will be from the indirect connection to the node $i$ to bring the utility value $\lambda_{l i}^{2}$, and this value is not included in the $u_{i j}$, the same way there are $u_{j l}>u_{i j} 0$,. However, due to $j l G$, which conflicts with the network stability.

To proof (2). In fact, if the two are not directly connected to any individual to add an edge, then each one of the at least two nodes benefit $\lambda_{i j}-\lambda_{i j}^{2}-c_{i j}$. When $c_{i j}<\lambda_{i j}-\lambda_{i j}^{2}$, then both sides will benefit. So in this context, only if the marketing network to become fully connected when the pair is stable.
To proof (3). First, we easily verify, when $\lambda_{i j}-\lambda_{i j}^{2}<c_{i j}<\lambda_{i j}$, the star structure marketing network is stable. Because $\lambda_{i j}-\lambda_{i j}^{2}<c_{i j}$, so add any non-central node between the two sides will make the benefits of each node $\lambda_{i j}-\lambda_{i j}^{2}-c_{i j}<0$. Similarly, because $c_{i j}<\lambda_{i j}$, So that delete any one side of star structure marketing network will make each node utility at least change $c_{i j}-\lambda_{i j}<0$. So the star structure marketing network is stable.

By contradiction proof (4). If a non-empty branches set up marketing network node 1 degree there is a connection, then the node connected with the side which called "hanging side." 。 "Hanging side" of the other end node will want to remove the edge, because it can increase the node utility $c_{i j}-\lambda_{i j}>0$. Also note that if the " hanging side " of both ends of the node degree is 1 , then remove the two sides after this node will become isolated nodes. Thus, the degree of any non-empty and stability network is more than 1. 1 .

\section{ACKNOWLEDGMENT}

This article funded by the research projects of Shaanxi Education Provincial Department ( 15JK2176) ), and xijing university research projects (XJ140204).

\section{REFERENCES}

[1] Yunlong Gao. design and management of enterprise marketing network [M] Beijing: Social Science Science Literature Publishing House, 2006.1-4.

[2] Xiaofan Wang, Xiang Li, Guanrong Chen. complex network theory and its application [M] Beijing: Tsinghua University Press, 2006.18-46.

[3] Barabási A L, Albert R. Emergence of Scaling in Random Networks [J]. Science,1999,286(5439):509-512.

[4] Matthew o. Jackson, Asher Wolinsky. A strategic model of social and economic networks[J]. Journal of economic theory, 1996(71):44-74.

[5] Y.M.Moon,P.Park,W.H.Kwon,Y.S.Lee. Delay-dependent robust stabilization of uncertain state-delayed systems[J],Int.J.Control,2001, 74(14):1447-1455.

[6] Xin Rui Zhang, Hengshan Wang. complex supply chain network model [J] Industrial Technology \& Economy, 2008 (2): 70-81.

[7] Chunfa Li, Shier Qi, Jian Li .supply chain network of dynamic equilibrium problem [J] Tianjin University, 2006 (6): 274-278

[8] Anna Nagurney, June Dong, Ding Zhang. A Supply Chain Network Equilibrium Model[J].Transportation Research,2012,38(5):281304.

[9] June Dong, Ding Zhang, Hong Yan, et al. Supply Chain Networks: Decision-Making Under Uncertainty [J]. Annals of Operations Research,2015,135(1):155-159.

[10] Lalonde B J. Supply Chain Management: Myth or Reality [J]. Supply Chain Management Review,20077, 3(1):6-7. 